\title{
Affleck-Dine inflation
}

\author{
James M. Cline $\odot$, Matteo Puel $\odot$, and Takashi Toma \\ McGill University, Department of Physics, 3600 University Street, Montréal, Quebec H3A2T8, Canada
}

(Received 22 October 2019; accepted 29 January 2020; published 19 February 2020)

\begin{abstract}
The Affleck-Dine mechanism in its simplest form provides baryogenesis from the out-of-equilibrium evolution of a complex scalar field with a simple renormalizable potential. We show that such a model supplemented by nonminimal coupling to gravity can also provide inflation, consistent with Planck constraints, simultaneously with the generation of the baryon asymmetry. The predictions of the model include significant tensor-to-scalar ratio and possibly baryon isocurvature fluctuations. The reheating temperature is calculable, making the model fully predictive. We require color triplet scalars for reheating and transferring the primordial baryon asymmetry to quarks; these could be observable at colliders. They can also be probed at higher scales by searches for quark compositeness in dijet angular distributions, and flavor-changing neutral current effects.
\end{abstract}

DOI: 10.1103/PhysRevD.101.043014

\section{INTRODUCTION}

Theoretical mechanisms for baryogenesis abound and take many very different forms, but one common attribute is that they occur at some cosmological epoch following inflation. This seems like a necessity, since exponential expansion should dilute any preexisting baryon asymmetry. Warm inflation provides an exception; see Ref. [1]. In this work we show that it is possible to generate the baryon asymmetry of the universe (BAU) during the course of ordinary cold inflation, if the inflaton carries baryon number.

One of the earliest proposed baryogenesis mechanisms was that of Affleck and Dine (AD) [2] in which a complex scalar field carrying baryon number can spontaneously create the BAU starting from field values displaced from the minimum of the potential. A baryon-violating coupling is required to satisfy Sakharov's requirements [3]. Although the AD mechanism is most commonly implemented in supersymmetric models whose potentials have nearly flat directions, it was originally demonstrated using a simple renormalizable potential of the form

$$
V_{J}=m_{\phi}^{2}|\phi|^{2}+\lambda|\phi|^{4}+i \lambda^{\prime}\left(\phi^{4}-\phi^{* 4}\right)
$$

in the seminal reference [2]. (We use the subscript to denote the Jordan frame since a change of frames will be invoked below.) When $\lambda^{\prime}=0$, the potential has a $\mathrm{U}(1)$ global

Published by the American Physical Society under the terms of the Creative Commons Attribution 4.0 International license. Further distribution of this work must maintain attribution to the author(s) and the published article's title, journal citation, and DOI. Funded by SCOAP ${ }^{3}$. symmetry, that we will identify with baryon number. A generic initial condition such that $\langle\phi\rangle \neq 0$ spontaneously breaks $C P$ and thermal equilibrium, as also required by Sakharov. The field winds around, at first generating baryon number, until Hubble damping of $\langle\phi(t)\rangle$ makes the $\lambda^{\prime}$ interaction negligible, and baryon number becomes conserved.

The same kind of potential could be used for a two-field version of chaotic inflation [4]. Constraints from the Planck experiment now disfavor chaotic inflation with $\phi^{2}$ or $\phi^{4}$ potentials [5] since they predict too high tensor-to-scalar ratio $r$, given the measured value of the scalar perturbation spectral index $n_{s}=0.965 \pm 0.004$. However this problem can be cured by adding a nonminimal coupling to gravity (we write $2 \xi$ rather than $\xi$ to agree with the usual convention for inflation along a single component of the complex scalar),

$$
\mathcal{L}_{J}=\frac{m_{P}^{2}}{2} R\left(1+2 \xi|\phi|^{2}\right)
$$

where $m_{P}=2.44 \times 10^{18} \mathrm{GeV}$ is the reduced Planck mass, that we set to 1 unless explicitly shown. This introduces a noncanonical kinetic term for $\phi$ upon Weyl transforming to the Einstein frame, and it flattens the potential at large field values to make the predictions of the model compatible with Planck observations, in the case of a real scalar field inflaton [6,7]. Our goal is to determine whether this can still hold true for the two-field model, while at the same time generating the observed baryon asymmetry. A potential issue is that isocurvature perturbations can be produced in two-field models, and these are constrained by the Planck observations. 
A similar idea was explored in Refs. [8,9], using flat directions in supergravity models as the inflaton. Models with simpler potentials, more similar to the one we consider, were studied in Refs. [10,11]. All of these previous studies are in the context of conventional chaotic inflation, and do not address the problem that the predictions of $r$ versus $n_{s}$ are excluded by Planck data. Isocurvature fluctuations are considered in Refs. [8,10]. We disagree with the predictions of Ref. [8], which do not take into account the decay of the entropy perturbation between horizon crossing and late times. The predictions of Ref. [10] for the power in entropy perturbations are consistent with ours, being below observable levels, but in the following we point out that the correlation between adiabatic and entropy modes can be large enough to be observed in current and upcoming CMB experiments.

\section{MODEL}

Equations (1) and (2) are sufficient to determine the inflationary trajectory until the epoch of reheating. It is convenient to make a Weyl rescaling of the metric, $g_{\mu \nu} \rightarrow \Omega^{2} g_{\mu \nu}$, with $\Omega^{2}=1 /\left(1+2 \xi|\phi|^{2}\right)$. The Lagrangian in the Einstein frame, including gravity, is then

$$
\mathcal{L}_{E}=\frac{1}{2} R+\Omega^{4}\left(\frac{|\partial \phi|^{2}}{\Omega^{2}}+3 \xi^{2}\left(\partial|\phi|^{2}\right)^{2}-V_{J}\right) .
$$

Writing the complex scalar as $\phi=(X+i Y) / \sqrt{2}$ and ignoring spatial gradients, the scalar kinetic term takes the form

$$
\mathcal{L}_{\text {kin }}=\frac{1}{2} \Omega^{2}\left(\dot{X}^{2}+\dot{Y}^{2}\right)+3 \Omega^{4} \xi^{2}(X \dot{X}+Y \dot{Y})^{2}
$$

with $\Omega^{2}=1 /\left(1+\xi\left(X^{2}+Y^{2}\right)\right)$. Thus $X$ and $Y$ are not canonically normalized fields. Instead of reexpressing them in terms of such fields, we will numerically solve the equations of motion for $X$ and $Y$ to determine the predictions for inflation and baryogenesis. Details of deriving the first-order equations convenient for numerical integration can be found for example in Ref. [12] [see Eqs. (2.100)-(2.101)]. We choose initial conditions close to the inflationary attractor solution, by setting the derivatives of the canonical momenta $\Pi_{X}=d \mathcal{L} / d \dot{X}, \Pi_{Y}=d \mathcal{L} / d \dot{Y}$ initially to zero.

More is needed in order to get reheating and transfer of the baryon asymmetry, initially stored in $\phi$, into quarks. A natural option for reheating is the Higgs portal coupling $\lambda_{\phi h}|\phi|^{2}|H|^{2}$. However since we also need a coupling to quarks, it is simpler to use the same interactions both for reheating and for transfer of the baryon asymmetry. This can be accomplished by introducing three QCD triplet scalars $\Phi_{i}$ carrying baryon number $2 / 3$, with couplings

$$
\begin{aligned}
V_{\Phi}= & \epsilon_{a b d}\left(\lambda^{\prime \prime} \phi^{*} \Phi_{1}^{a} \Phi_{2}^{b} \Phi_{3}^{d}+y_{1} \Phi_{1}^{a} \bar{u}_{R}^{b} d_{R}^{c, d}\right. \\
& \left.+y_{2} \Phi_{2}^{a} \bar{u}_{R}^{b} d_{R}^{c, d}+y_{3} \Phi_{3}^{a} \bar{d}_{R}^{b} d_{R}^{c, d}\right)+ \text { H.c. }
\end{aligned}
$$

where $a, b, d$ are color indices, the quarks are right-handed [SU $(2)_{L}$ singlets] and $d_{R}^{c}$ denotes the conjugate down-type quark. For simplicity we omit generation indices on the quarks and the Yukawa couplings $y_{i}$. These interactions allow for the decay $\phi \rightarrow$ uudddd via virtual $\Phi_{i}$ exchange, and imply that $\phi$ carries baryon number 2 . The same conclusion holds if we choose $\Phi_{1} \bar{u}_{R} u_{R}^{c}$ and $\Phi_{2,3} \bar{d}_{R} d_{R}^{c}$ couplings instead of (5).

For small values of the $\lambda^{\prime \prime}$ coupling, we can view reheating as occurring through the perturbative decays $\phi \rightarrow \Phi_{1} \Phi_{2} \Phi_{3}$, which rapidly thermalize with the quarks and thereby the rest of the standard model degrees of freedom. Assuming that $\phi$ is much heavier than $\Phi_{i}$, the decay rate is

$$
\Gamma_{\phi}=\frac{3 \lambda^{\prime \prime 2}}{256 \pi^{3}} m_{\phi}
$$

\section{INFLATION + BARYOGENESIS}

An interesting aspect of our model is that the same parameters that influence inflationary observables can also affect the magnitude of the baryon asymmetry. Thus, although we describe the two processes separately, a fully viable model depends upon the interplay between the two.

\section{A. Slow-roll parameters}

Although we can solve for the inflaton trajectories without reference to the canonically normalized fields, that we will denote by $(U, V)$, it is necessary to know them for computing inflationary perturbations. It is straightforward to diagonalize the kinetic term (4) at a given point in field space to find

$$
\begin{aligned}
\left(\begin{array}{c}
\dot{X} \\
\dot{Y}
\end{array}\right) & =\left(\begin{array}{cc}
c_{\theta} & -s_{\theta} \\
s_{\theta} & c_{\theta}
\end{array}\right)\left(\begin{array}{cc}
e_{1} & 0 \\
0 & e_{2}
\end{array}\right)\left(\begin{array}{cc}
c_{\psi} & s_{\psi} \\
-s_{\psi} & c_{\psi}
\end{array}\right)\left(\begin{array}{c}
\dot{U} \\
\dot{V}
\end{array}\right) \\
& \equiv Z_{0} R_{\psi}\left(\begin{array}{c}
\dot{U} \\
\dot{V}
\end{array}\right) \equiv Z\left(\begin{array}{c}
\dot{U} \\
\dot{V}
\end{array}\right)
\end{aligned}
$$

with $e_{1}=\Omega^{-1}\left(1+6 \Omega^{2} \xi^{2}\left(X^{2}+Y^{2}\right)\right)^{-1 / 2}, e_{2}=\Omega^{-1}, \theta=$ $\tan ^{-1}(Y / X)$. Then $\mathcal{L}_{\text {kin }}=\frac{1}{2}\left(\dot{U}^{2}+\dot{V}^{2}\right)$. The matrix $Z$ allows us to transform slow-roll parameters computed in the original field basis (indices $i, j$ ) to those in the canonical basis (indices $m, n$ ):

$$
\begin{aligned}
\epsilon_{m} & =\frac{\left(Z_{i m} \partial_{i} V_{E}\right)^{2}}{2 V_{E}^{2}}, \\
\eta_{m n} & =Z_{i m} Z_{j n} \frac{\partial_{i} \partial_{j} V_{E}}{V_{E}}+Z_{i m} \partial_{i} Z_{j n} \frac{\partial_{j} V_{E}}{V_{E}}
\end{aligned}
$$

where $V_{E}=\Omega^{4} V_{J}$ is the Einstein frame potential. 
TABLE I. Parameters and initial values for two benchmark models, including the total number of $e$-foldings of inflation $N_{\text {tot }}$, number of $e$-foldings before horizon crossing $N_{*}$, spectral index $n_{s}$ (evaluated at $k_{*}=0.05 \mathrm{Mpc}^{-1}$ ), tensor-to-scalar ratio $r$ and off-diagonal transfer matrix element $T_{R S}$, which is a measure of the correlation between adiabatic and isocurvature perturbations.

\begin{tabular}{lcccccccccccc}
\hline \hline Model & $m_{\phi} / m_{P}$ & $\lambda$ & $\lambda^{\prime}$ & $\xi$ & $\lambda^{\prime \prime}$ & $X_{0}$ & $Y_{0}$ & $N_{\text {tot }}$ & $N_{*}$ & $n_{s}$ & $r$ & $T_{R S}$ \\
\hline 1 & $6.43 \times 10^{-7}$ & $8.73 \times 10^{-12}$ & $6.89 \times 10^{-13}$ & $5.96 \times 10^{-2}$ & $8.67 \times 10^{-5}$ & 18.4 & 6.63 & 65 & 53.1 & 0.962 & $1.4 \times 10^{-2}$ & $9 \times 10^{-2}$ \\
2 & $4.67 \times 10^{-7}$ & $3.49 \times 10^{-11}$ & $6.68 \times 10^{-13}$ & 0.180 & $2.93 \times 10^{-5}$ & 23.7 & 0.91 & 146 & 52.0 & 0.961 & $7.2 \times 10^{-3}$ & $4 \times 10^{-5}$ \\
\hline \hline
\end{tabular}

The extra rotation $R_{\psi}$ in Eq. (7) is not necessary for diagonalizing the kinetic term, but it is required in order to be able to interpret $Z$ as the Jacobian matrix $\partial(X, Y) /$ $\partial(U, V)$. If we omit $R_{\psi}$ so that $Z=Z_{0}$, such an interpretation is not generally consistent since then the relations

$$
\begin{aligned}
& U_{, Y X}=\partial_{X}\left(Z_{0}^{-1}\right)_{12}=\partial_{Y}\left(Z_{0}^{-1}\right)_{11}=U_{, X Y}, \\
& V_{, Y X}=\partial_{X}\left(Z_{0}^{-1}\right)_{22}=\partial_{Y}\left(Z_{0}^{-1}\right)_{21}=V_{, X Y}
\end{aligned}
$$

may not be satisfied. We are free to set $\psi=0$ at a given point in field space, such as the point of horizon crossing, but not its derivatives. Equations (9) with $Z_{0} \rightarrow Z_{0} R_{\psi}$ imply

$$
\begin{aligned}
& \left(Z_{0}^{-1}\right)_{22} \psi_{, X}-\left(Z_{0}^{-1}\right)_{21} \psi_{, Y}=\left(Z_{0}^{-1}\right)_{12, X}-\left(Z_{0}^{-1}\right)_{11, Y}, \\
& \quad-\left(Z_{0}^{-1}\right)_{12} \psi_{, X}+\left(Z_{0}^{-1}\right)_{11} \psi_{, Y}=\left(Z_{0}^{-1}\right)_{22, X}-\left(Z_{0}^{-1}\right)_{21, Y}
\end{aligned}
$$

This has the solution

$$
\begin{aligned}
\left(\begin{array}{c}
\psi_{, X} \\
\psi_{, Y}
\end{array}\right) & =\operatorname{det} Z_{0}\left(Z_{0}^{-1}\right)^{T}\left(\begin{array}{cc}
\left(Z_{0}^{-1}\right)_{12, X} & -\left(Z_{0}^{-1}\right)_{11, Y} \\
\left(Z_{0}^{-1}\right)_{22, X} & -\left(Z_{0}^{-1}\right)_{21, Y}
\end{array}\right) \\
& =\Omega^{2} \frac{e_{1} e_{2}}{X^{2}+Y^{2}}\left(\begin{array}{c}
-Y \\
X
\end{array}\right) .
\end{aligned}
$$

The consistent identification of $Z$ with a Jacobian matrix ensures that $\eta_{m n}$ is symmetric in $m n$, even though the second term in (8) is not explicitly symmetric. Then we can write the second term in Eq. (8) as

$$
\partial_{i} Z=\left(\partial_{i} Z_{0}\right) R_{\psi}+\psi_{, i} Z_{0} \partial_{\psi} R_{\psi}
$$

To compute the adiabatic perturbation spectrum and the tensor-to-scalar ratio, we use the slow-roll formalism of Ref. [13], evaluating the slow-roll parameters (8) along the numerically determined inflationary solutions. This requires going from the $U, V$ basis of the canonical fields to the $\sigma, s$ basis of adiabatic/entropy directions defined by

$$
\begin{aligned}
& d \sigma=c_{\alpha} d U+s_{\alpha} d V, \\
& d s=-s_{\alpha} d U+c_{\alpha} d V
\end{aligned}
$$

with $\alpha=\tan ^{-1}(\dot{V} / \dot{U})$. The rotated slow-roll parameters are given by [14]

$$
\begin{aligned}
\epsilon_{\sigma} & =\left(c_{\alpha} \partial_{U} V_{E}+s_{\alpha} \partial_{V} V_{E}\right)^{2} /\left(2 V_{E}^{2}\right), \\
\epsilon_{s} & \cong 0 \\
\eta_{\sigma \sigma} & =c_{\alpha}^{2} \eta_{U U}+2 c_{\alpha} s_{\alpha} \eta_{U V}+s_{\alpha}^{2} \eta_{V V}, \\
\eta_{s s} & =s_{\alpha}^{2} \eta_{U U}-2 c_{\alpha} s_{\alpha} \eta_{U V}+c_{\alpha}^{2} \eta_{V V} \\
\eta_{\sigma s} & =c_{\alpha} s_{\alpha}\left(\eta_{V V}-\eta_{U U}\right)+\left(c_{\alpha}^{2}-s_{\alpha}^{2}\right) \eta_{U V} .
\end{aligned}
$$

Then to leading order in the slow-roll expansion, the scalar spectral index and tensor-to-scalar ratio are [13]

$$
\begin{aligned}
n_{s} & =1-\left(6-4 c_{\Delta}^{2}\right) \epsilon_{\sigma}+2 s_{\Delta}^{2} \eta_{\sigma \sigma}+4 s_{\Delta} c_{\Delta} \eta_{\sigma s}+2 c_{\Delta}^{2} \eta_{s s}, \\
r & =16 \epsilon_{\sigma}
\end{aligned}
$$

where $c_{\Delta}=-2 \mathcal{C} \eta_{\sigma s}, s_{\Delta}=+\sqrt{1-c_{\Delta}^{2}}, \mathcal{C}=2-\ln 2-\gamma \cong$ 0.73 ( $\gamma$ is the Euler constant), and the derivatives of $V_{E}$ with respect to $U$ and $V$ are computed similarly to Eq. (8). Including the effect of isocurvature modes $\left(T_{R S}\right)$, which we will explain below, the scalar amplitude is

$A_{s}=\frac{V_{*}}{24 \pi^{2} \epsilon_{\sigma}}\left[1-2 \epsilon_{\sigma}+2 \mathcal{C}\left(3 \epsilon_{\sigma}-\eta_{\sigma \sigma}-2 \eta_{\sigma s} T_{R S}\right)\right]$

with $V_{*}=V_{E}$ evaluated at horizon crossing and we have neglected terms of order $T_{R S}^{2}$.

We searched the parameter space via Markov chain Monte Carlo (MCMC) to find models in agreement with Planck constraints on $A_{s}, n_{s}, r$ and the baryon asymmetry (discussed below). Two benchmark models are identified in Table I. The correlation of $r$ with $n_{s}$ is shown over the interval $N_{*}=(50,60) e$-foldings, for several values of $\xi$ and fixed values of the potential parameters corresponding to the two benchmark models in Fig. 1.

On each curve a heavy dot is indicated to show the prediction of the model, for the chosen value of $\lambda^{\prime \prime}$, that determines the reheating temperature and thus the number of $e$-foldings $N_{*}$ between horizon crossing and the end of inflation. The value of $N_{*}$ is determined by solving Eq. (47) of Ref. [5] (see also Ref. [15]),

$N_{*}=67-\ln \left(\frac{k_{*}}{H_{0}}\right)+\frac{1}{4} \ln \left(\frac{V_{*}^{2}}{m_{P}^{4} \rho_{\text {end }}}\right)+\frac{1}{12} \ln \left(\frac{\rho_{\mathrm{rh}}}{g_{*} \rho_{\mathrm{end}}}\right)$

where $H_{0}$ is the Hubble constant today, $\rho_{\text {end }}$ is the energy density at the end of inflation, $g_{*}=106.75+18$ (counting 

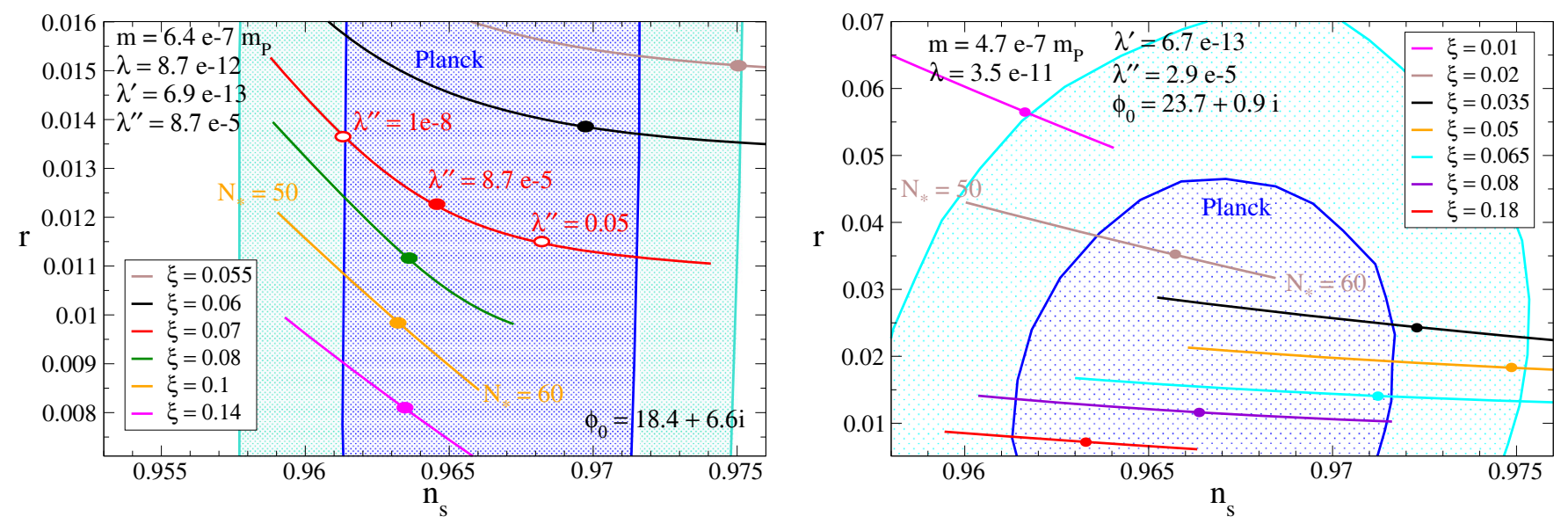

FIG. 1. Scalar-to-tensor ratio versus spectral index for several values of the nonminimal coupling $\xi$, varied around the parameters of models 1 (left) and 2 (right) given in Table I. The pivot scale is $k_{*}=0.002 \mathrm{Mpc}^{-1}$ for comparison with the Planck $1 \sigma$ and $2 \sigma$ allowed regions. The number of $e$-foldings between horizon crossing and the end of inflation, $N_{*}$, is allowed to vary between 50 and 60 , but the definite values shown by the solid dots are predicted by making a specific choice of $\lambda^{\prime \prime}$. The dependence on $\lambda^{\prime \prime}$ is shown on the $\xi=0.07$ curve for model 1 .

the extra degrees of freedom from the colored scalars), and the reference scale $k_{*}=0.002 \mathrm{Mpc}^{-1}$ for comparison with the Planck preferred regions in the $n_{s}-r$ plane. The energy density at the time of reheating is $\rho_{\mathrm{rh}}=\frac{4}{3} \Gamma_{\phi}^{2} m_{P}^{2}$, as explained below-see Eq. (24); this makes $N_{*}$ depend upon $\lambda^{\prime \prime}$ as $N_{*} \sim \frac{1}{3} \ln \lambda^{\prime \prime}$. Since $V_{*}$ appears in Eq. (16) but also depends upon $N_{*}$, we rescale the parameters of the potential while iteratively determining $N_{*}$, keeping $A_{s}=$ $e^{3.044} \times 10^{-10}$ fixed to the observed central value [5]. To illustrate the dependence on $\lambda^{\prime \prime}$, we indicate two other horizon-crossing positions on the $\xi=0.07$ curve of model 1 , for larger and smaller values of $\lambda^{\prime \prime}$. The relation between $\lambda^{\prime \prime}$ and the reheat temperature will be discussed in Sec. III C.

The strong correlation between the tensor ratio $r$ and the nonminimal coupling $\xi$ is also clearly seen in the larger

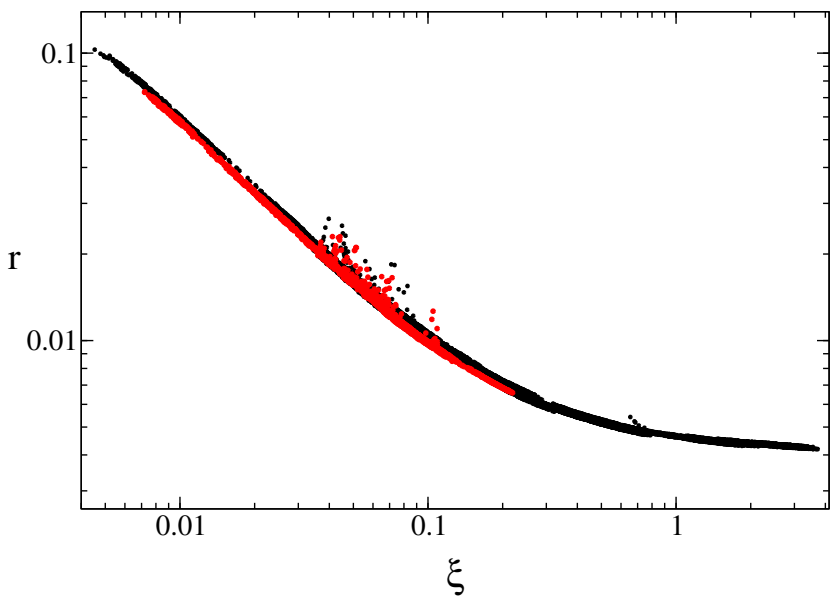

sample of models from two MCMC chains, Fig. 2 (left). The points shown have a total $\chi^{2}<10$, defining $\chi^{2}$ in the usual way in terms of the observables $r, n_{s}$ and $\eta_{B}$,

$$
\chi^{2}=\sum_{i} \frac{\left(x_{i}-\bar{x}_{i}\right)^{2}}{\delta x_{i}^{2}}
$$

summed over observables $x_{i}$ with central value $\bar{x}_{i}$ and experimental error $\delta x_{i}$. The black points come from a chain where the experimental limit on $r$ was somewhat relaxed. The correlation between $r$ and $n_{s}$ within the chains is also notable, as shown in Fig. 2 (right). In both plots, one can notice a population of models scattered away from the main trends. These are special cases in which the total number of $e$-foldings of inflation are not much greater than the minimum required, $N_{e} \sim 60$. We will discuss these cases in more detail below.

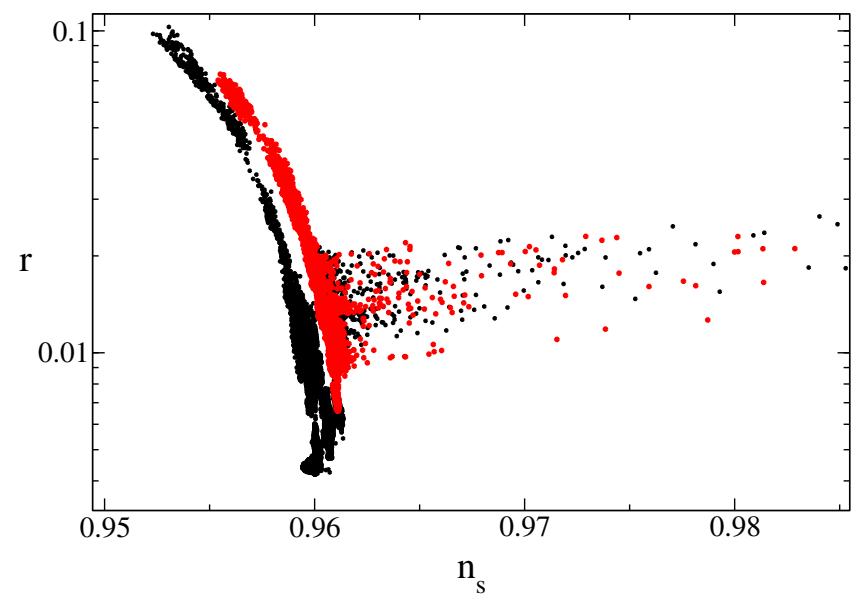

FIG. 2. Scatter plots from the MCMC search of parameter space. Left: correlation of $r$ with $\chi$. Right: correlation of $r$ (evaluated at $k_{*}=0.002 \mathrm{Mpc}^{-1}$ ) with $n_{s}$ (at $k_{*}=0.05 \mathrm{Mpc}^{-1}$ ). Black versus red points correspond to two different chains as described in the text. 


\section{B. Isocurvature fluctuations}

During inflation, the components of the canonically normalized fields $U, V$ can fluctuate by order $H /(2 \pi)$, where $H$ is the Hubble parameter. Fluctuations $d \sigma$ normal to the inflaton trajectory are entropy modes, and they could become observable isocurvature fluctuations if they decay into different species than do the adiabatic fluctuations, that are parallel to the trajectory. The relation between adiabatic/ entropy perturbations and the canonical field fluctuations is given in Eq. (12).

To find the observable entropy fluctuations, we need to compare $(d \sigma, d s)$ to the directions in field space that correspond to baryon number fluctuations $d B$, and the orthogonal direction, that will be related to $(d U, d V)$ through some different rotation angle $\beta$. Numerically we find that $\beta \cong 0$ during inflation, implying that the entropy perturbations are purely in the baryon number (compensated by radiation) to a good approximation, known as BDI (baryon density isocurvature). This can be seen starting from the definition of baryon density from the zeroth component of the baryon current carried by $\phi$,

$$
n_{B}=j_{B}^{0}=-2 i\left(\phi \dot{\phi}^{*}-\phi^{*} \dot{\phi}\right)=2(Y \dot{X}-X \dot{Y})
$$

leading to the fluctuation

$$
\delta n_{B}=2(\dot{X} \delta Y-\dot{Y} \delta X)+\ldots
$$

where the omitted terms are subleading in the slow-roll approximation. The direction of the fluctuation (19) turns out numerically to be very nearly orthogonal to the inflaton trajectory in field space. Although both $\sigma$ and $s$ decay into quarks during reheating, only $s$ decays encode the baryon asymmetry, whereas $\sigma$ decays equally into quarks and antiquarks, that thermalize with the rest of the standard model degrees of freedom.

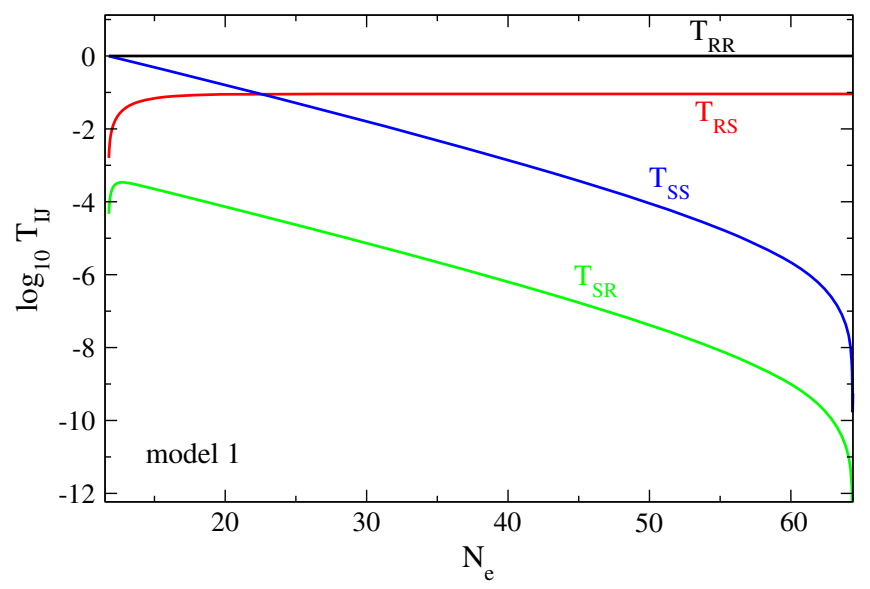

We closely follow the formalism of Ref. [13] (see also Ref. [14]) to compute the power in isocurvature. The main task is to numerically solve the equations for the evolution of the perturbations $d U, d V$ between horizon crossing and the end of inflation,

$$
\begin{aligned}
d U^{\prime \prime}= & -C_{1} d U^{\prime}-3\left(\bar{\eta}_{U U} d U+\bar{\eta}_{U V} d V\right)+U^{\prime} d C \\
& +\left(U^{\prime 2}\right)^{\prime} d U+\left(U^{\prime} V^{\prime}\right)^{\prime} d V \\
d V^{\prime \prime}= & -C_{1} d V^{\prime}-3\left(\bar{\eta}_{V V} d V+\bar{\eta}_{U V} d U\right)+V^{\prime} d C \\
& +\left(V^{\prime 2}\right)^{\prime} d V+\left(U^{\prime} V^{\prime}\right)^{\prime} d U
\end{aligned}
$$

and to relate them to the adiabatic/isocurvature perturbations $d \sigma, d s$ using Eq. (12). Here primes denote $d / d N_{e}$, $C_{1}=3+H^{\prime} / H$, and $d C=C_{1}\left(U^{\prime} d U+V^{\prime} d V\right)$. The barred parameters $\bar{\eta}_{i j}$ are defined as in Eq. (8), except that we divide by the total energy density $\rho=3 H^{2}$ instead of $V_{E}$, so that the equations remain valid even when the slow-roll approximation is not.

The transfer function for the curvature (adiabatic) and entropy perturbations is a matrix

$$
\left(\begin{array}{ll}
T_{R R} & T_{R S} \\
T_{S R} & T_{S S}
\end{array}\right)
$$

that relates the amplitudes of $(d \sigma, d s)$ at horizon crossing to those at a later time, after inflation. We can get the matrix elements by solving the system (20) from the respective initial conditions $(d \sigma, d s)=(1,0)$ and $(0,1)$. The results are shown for the two benchmark models in Fig. 3. The adiabatic perturbation is conserved, resulting in $T_{R R}=1$, and the $T_{S R}$ element is always very small, in accordance with the slow-roll prediction $T_{S R}=0$ [13], meaning that there is negligible conversion of entropy to adiabatic modes.

Numerically it is difficult to evolve the transfer matrix deep into the postinflationary phase, because of the fast oscillations of the fields. However since the solutions

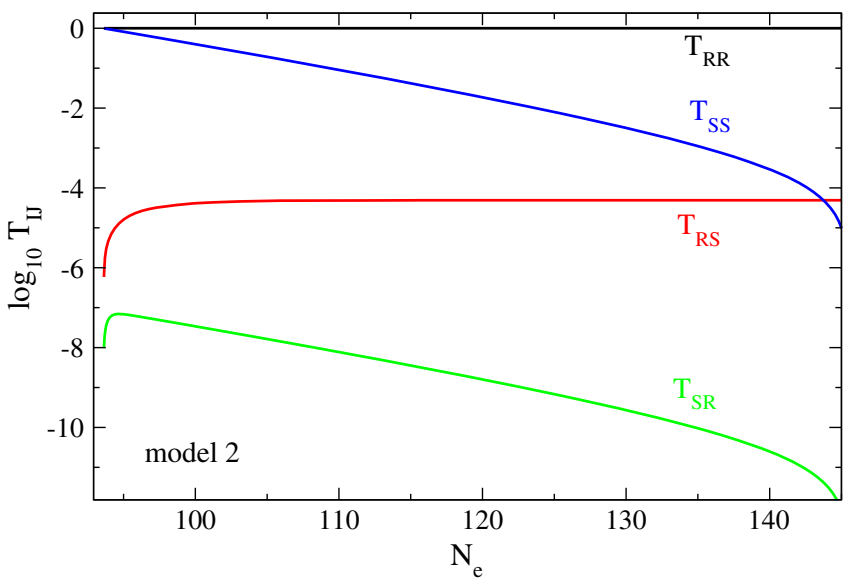

FIG. 3. Evolution of transfer matrix elements for the adiabatic and isocurvature perturbations, versus number of $e$-foldings, for models 1 and 2 from Table I. 
become quite smooth at this point, it seems reasonable to extrapolate them into the reheating era. Hence we have assumed that $T_{R R}$ and $T_{R S}$ continue to stay constant, and that $T_{S R}$ and $T_{S S}$ remain negligibly small, as Fig. 3 suggests. Once the inflaton decays, both adiabatic and entropy modes (which are approximately aligned with the modulus and phase of $\phi$ ) will decay into quarks and antiquarks. The only difference between the two modes is that the isocurvature modes are also correlated with baryon number, which is conserved by this time, so that the baryon asymmetry encoded in $s$ is preserved during the decays.

For all cases in our MCMC, the entropy autocorrelation $T_{S S} \ll 0.1$ is too small to be observable, but in some cases like in model 1, the cross-correlation $T_{R S}$ is significant. It is related to the correlation angle, which to leading order in slow-roll parameters is given by [13]

$$
\cos \Delta=\frac{T_{R S}}{\sqrt{1+T_{R S}^{2}}}
$$

which is constrained by Planck as $|\cos \Delta| \lesssim 0.1-0.3$, depending upon pivot scale $k_{*}$ and which datasets are combined. (Reference [5] notes that the constraints on BDI correlation are the same as for cold dark matter isocurvature.) Therefore model 1 is an example where the predicted BDI correlation is close to the experimental sensitivity.

The models with large BDI require somewhat special initial conditions, in which the total duration of inflation is not more than $\sim 80 e$-foldings. This is because significant curvature of the inflaton path in field space is needed during horizon crossing for generating isocurvature. Models with long periods of inflation tend to have such curvature earlier than horizon crossing, subsequently becoming nearly linear and thus resembling single-field inflation. This is illustrated for the two benchmark models in Fig. 4, that shows the field trajectories and horizon-crossing points. It is further borne out by Fig. 5 showing the correlation between $\left|T_{R S}\right|$ and total number of $e$-foldings $N_{\text {tot }}$ for models within a MCMC

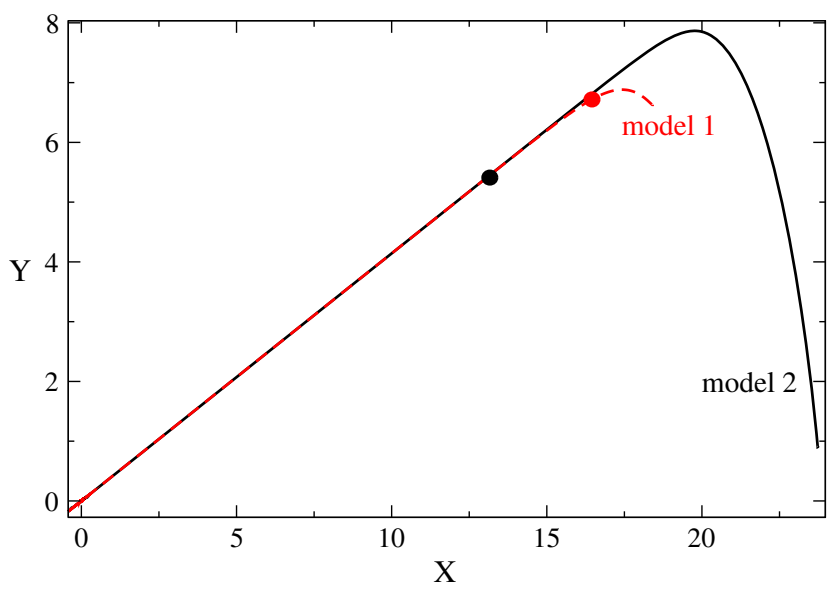

FIG. 4. Inflaton trajectories in field space for the benchmark models. Horizon crossing is indicated by the heavy dot.

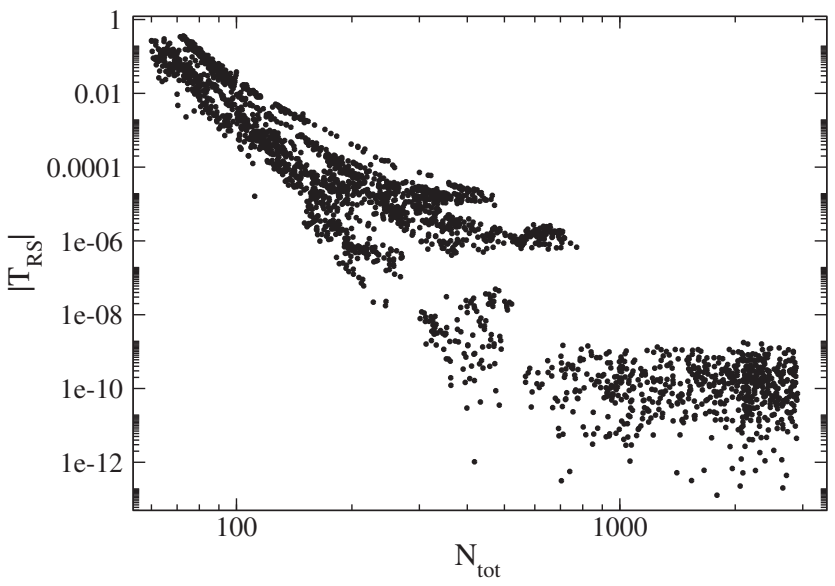

FIG. 5. Scatter plot of isocurvature correlation $\left|T_{R S}\right|$ versus the total number of $e$-foldings of inflation $N_{\text {tot }}$ from the MCMC.

chain satisfying $\chi^{2}<10$. On the other hand, models like our benchmark model 2, having longer periods of inflation, lead to predictions that are relatively insensitive to the initial conditions, since the field trajectory settles into a unique trough in the potential.

\section{Baryogenesis and reheating}

To compute the baryon asymmetry, we use the baryon density stored in $\phi$, Eq. (18). It is convenient to compare this to the number density of $\phi$ particles, prior to reheating,

$$
n_{\phi}=\frac{\rho_{\phi}}{m_{\phi}}
$$

since the ratio $\eta=n_{B} / n_{\phi}$ reaches a constant value that we denote as $\eta_{e}$ at the end of inflation, during the period of $\phi$ oscillations around the minimum of the potential. The time evolution of $\eta$ is illustrated for model 1 in Fig. 6.

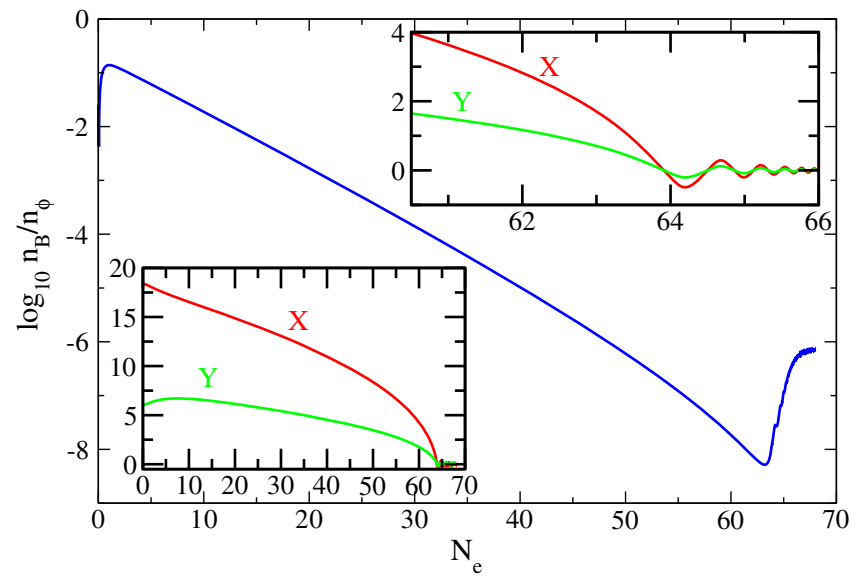

FIG. 6. Baryon-to-inflaton ratio during inflation and shortly after its end, versus number of $e$-foldings $N_{e}$, for benchmark model 1. Insets show the evolution of the field components, $\phi=(X+i Y) / \sqrt{2}$. 
Reheating occurs at the time $t_{\mathrm{rh}}=1 / \Gamma_{\phi}$ where $\Gamma_{\phi}$ is the decay width of $\phi$. Defining $n_{\phi}=n_{\phi, e}$ at the end of inflation $\left(t=t_{e}\right), n_{\phi}$ at the time of reheating will be

$$
\begin{aligned}
n_{\phi, \mathrm{rh}} & =n_{\phi, e}\left(\frac{a_{e}}{a_{\mathrm{rh}}}\right)^{3}=\frac{n_{\phi, e}}{\left(1+\frac{3}{2} \sqrt{\frac{m_{\phi} n_{\phi, e}}{3 m_{P}^{2}}}\left(t_{\mathrm{rh}}-t_{e}\right)\right)^{2}} \\
& \cong \frac{4 m_{P}^{2} \Gamma_{\phi}^{2}}{3 m_{\phi}}
\end{aligned}
$$

where we used the fact that the $\phi$ oscillations matter dominate the Universe until reheating, and $t_{\mathrm{rh}} \gg t_{e}$. The value of $n_{\phi, \mathrm{rh}}$ is independent of $n_{\phi, e}$, so long as the latter is large enough to provide sufficient expansion of the Universe prior to reheating. This will be true if the energy density at the end of inflation is much greater than that at reheating.

The baryon-to-entropy ratio at reheating is given by

$$
\eta_{B}=\eta_{e} \frac{n_{\phi, \mathrm{rh}}}{s}
$$

with $s=\left(2 \pi^{2} / 45\right) g_{*} T_{\mathrm{rh}}^{3}$ and reheat temperature [16]

$$
\begin{aligned}
T_{\text {rh }} & =\left(\frac{90}{\pi^{2} g_{*}}\right)^{1 / 4}\left(\Gamma_{\phi} m_{P}\right)^{1 / 2} \\
& =1.7 \times 10^{14} \mathrm{GeV}\left(\frac{\lambda^{\prime \prime}}{10^{-2}}\right)\left(\frac{m_{\phi} / m_{P}}{5 \times 10^{-7}}\right)^{1 / 2} .
\end{aligned}
$$

Including a factor of 36/111 [17] for the reduction of baryon number by redistribution into lepton number by sphalerons, it follows that

$$
\eta_{B} \cong 6.1 \times 10^{-4} \eta_{e} \lambda^{\prime \prime}\left(\frac{m_{P}}{m_{\phi}}\right)^{1 / 2}
$$

which is conserved into the late Universe. The measured value is $\eta_{B}=8.6 \times 10^{-11}$ [18].

The coupling $\lambda^{\prime \prime}$ should be small in order to justify the perturbative reheating assumption, but from the point of view of technical naturalness, it need not be very small. A three-loop diagram involving $\lambda^{\prime \prime}$ renormalizes the $\lambda|\phi|^{4}$ interaction, giving the estimate

$$
\lambda^{\prime \prime} \lesssim\left(16 \pi^{2}\right)^{3 / 4}(\lambda / 36)^{1 / 4} \cong 0.05
$$

to avoid destabilizing the inflationary potential by quantum corrections.

The baryon asymmetry generated during inflation depends sensitively on the value of the $B$-violating coupling $\lambda^{\prime}$. In Fig. 7 we again show how $\eta_{B}$ evolves with $N_{e}$ from the beginning of inflation until shortly after it ends, but for a range of different values of the baryon-violating coupling $\lambda^{\prime}$. The effect of the $\phi$ oscillations can be seen

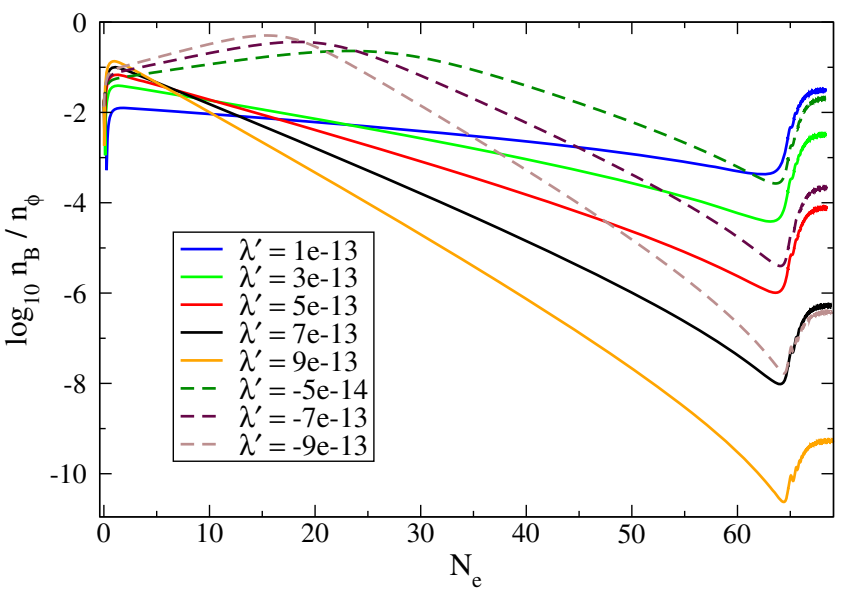

FIG. 7. Baryon-to-inflaton ratio during inflation and shortly after its end, versus number of $e$-foldings $N_{e}$, for several values of $\lambda^{\prime}$. Other potential parameters are fixed at those of model 1 . The curves are in the same order as the key, from top to bottom at late times. Positive values of $\lambda^{\prime}$ are shown with solid curves, negative with dashed.

briefly around $N_{e}=65$, but these are quickly Hubble damped and $\eta_{B}$ settles to a constant value that we have identified as $\eta_{e}$ in Eq. (25). The dependence of the final baryon asymmetry is not monotonic. At first this may be surprising, since one can derive the time dependence of $n_{B}$ from the inflaton field equations,

$$
\begin{aligned}
\dot{n}_{B} & =2 i\left(\phi^{*} \ddot{\phi}-\ddot{\phi}^{*} \phi\right) \\
& =-3 H n_{B}-8 \lambda^{\prime}\left(\phi^{4}+\phi^{* 4}\right) .
\end{aligned}
$$

However one finds that $\lambda^{\prime}$ has an important effect on the background inflaton trajectory, which explains the nonlinear dependence. This is illustrated in Fig. 8. Hence the processes of inflation and baryogenesis are nontrivially

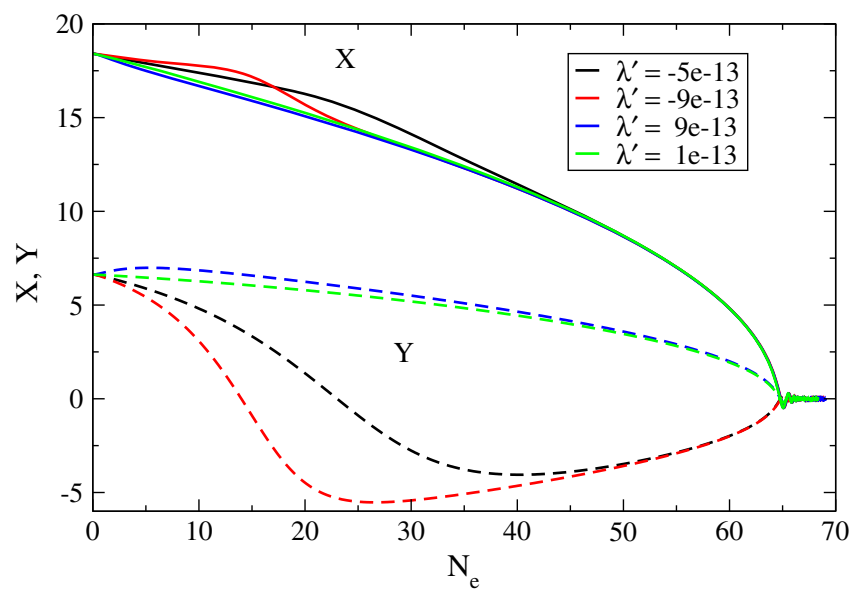

FIG. 8. Inflaton trajectories $X\left(N_{e}\right)$ (solid) and $Y\left(N_{e}\right)$ (dashed) for four different values of the baryon-violating coupling $\lambda^{\prime}$. Other parameters are fixed to those of model 1. 
TABLE II. Lower limits from meson-antimeson mixing on parameters entering the Wilson coefficients of fourquark operators from integrating out the heavy color triplet $\Phi_{3}$.

\begin{tabular}{lcccc}
\hline \hline System & $K^{0}-\bar{K}^{0}$ & $K^{0}-\bar{K}^{0}$ & $B^{0}-\bar{B}^{0}$ & $B_{s}^{0}-\bar{B}_{s}^{0}$ \\
Coefficient & $m_{\Phi_{3}} \operatorname{Re}\left[y_{3, d d} y_{3, s s}^{*}\right]^{-1 / 2}$ & $m_{\Phi_{3}} \operatorname{Im}\left[y_{3, d d} y_{3, s s}^{*}\right]^{-1 / 2}$ & $m_{\Phi_{3}}\left|y_{3, d d} y_{3, b b}^{*}\right|^{-1 / 2}$ & $m_{\Phi_{3}}\left|y_{3, s s} y_{3, b b}^{*}\right|^{-1 / 2}$ \\
Limit & $1.1 \times 10^{3} \mathrm{TeV}$ & $2.1 \times 10^{4} \mathrm{TeV}$ & $990 \mathrm{TeV}$ & $245 \mathrm{TeV}$ \\
\hline \hline
\end{tabular}

intertwined in our model: Adjusting $\lambda^{\prime}$ can affect not only $\eta_{B}$ but also the inflationary observables.

The effects of $B$ violation after inflation are negligible. At low energies, integrating out $\phi$ and $\Phi_{i}$ leads to a dimension-36 operator involving 24 quarks. It could induce conversion of four neutrons into their antiparticles in a neutron star, but the rate is far too small to be significant. In the early Universe, we must check that the $\Delta B=8, \Phi_{i}^{12}$ operator induced by $\phi$ exchange is out of equilibrium, to avoid washing out the $B$ asymmetry. The rate can be estimated as

$$
\Gamma_{\Delta B=8} \sim \frac{\lambda^{12} \lambda^{\prime \prime 8} T^{17}}{m_{\phi}^{16}} \lesssim \frac{T^{2}}{m_{P}}
$$

By demanding that the decoupling temperature exceed the reheat temperature $T_{\mathrm{rh}}$ in Eq. (26), we find a constraint

$$
\lambda^{\prime \prime} \lesssim 20 \frac{m_{\phi}^{17 / 46}}{\left(\lambda^{\prime}\right)^{2 / 23}} \sim 1.5
$$

which is more lenient than the consistency requirement (28).

\section{PARTICLE PHYSICS IMPLICATIONS}

The colored scalars $\Phi_{i}$ can have observable effects at low energies. If sufficiently light, they can be pair produced at the LHC. The Yukawa interactions in Eq. (5) have the same form as $R$-parity violating coupling of squarks to quarks in supersymmetric models, leading to various mass exclusions in the range $80-525 \mathrm{GeV}$ [19] or $100-600 \mathrm{GeV}$ [20], depending upon the flavor structure of the couplings.

However heavier colored scalars can be probed indirectly, using an effective field theory description where they are integrated out to give dimension-six, four-quark operators. For baryogenesis, the flavor structure of the new Yukawa couplings was not important, but at low energies it can have an observable effect on the angular distributions of jets at the LHC, or flavor-changing neutral currents like meson-antimeson oscillations. Using chiral Fierz identities [21], the effective Lagrangian is

$$
\begin{aligned}
\mathcal{L}= & -\sum_{A=1,2} \delta_{b d}^{a e} \frac{y_{A, i j} y_{A, k l}^{*}}{2 m_{\Phi_{A}}^{2}}\left(\bar{u}_{i}^{a} \gamma^{\mu} P_{R} u_{k, b}\right)\left(\bar{d}_{j}^{e} \gamma_{\mu} P_{R} d_{l, d}\right) \\
& -\frac{y_{3, i i} y_{3, j j}^{*}}{m_{\Phi_{3}}^{2}}\left(\bar{d}_{i}^{a} \gamma^{\mu} P_{R} d_{j, a}\right)\left(\bar{d}_{i}^{b} \gamma_{\mu} P_{R} d_{j, b}\right)
\end{aligned}
$$

where $a, b, d, e$ are color indices, $i, j, k, l$ label flavor, and $P_{R}$ projects onto right-handed chirality. In the bottom line we have specialized to the case where $i \neq j$ and the operator contributes to meson-antimeson oscillations, since these combinations are much more severely constrained than the flavor-diagonal ones, or those connecting mesons of different masses.

From dijet angular distributions, the CMS Collaboration finds a limit of [22]

$$
\left(\frac{m_{\Phi}^{2}}{y y^{*}}\right)^{1 / 2} \gtrsim 7 \mathrm{TeV}
$$

for flavor-diagonal operators, presumably of the first generation (since the limit on higher-generation quarks will be somewhat weakened by parton distribution functions). However $K^{0}-\bar{K}^{0}, B^{0}-\bar{B}^{0}$ and $B_{s}^{0}-\bar{B}_{s}^{0}$ mixing give more stringent constraints [23] shown in Table II.

\section{CONCLUSIONS}

We have studied a new model of inflation with the novel feature that the inflaton carries baryon number, and it can produce the baryon asymmetry via the Affleck-Dine mechanism, mostly during inflation, with relatively small evolution over the few $e$-foldings after inflation ends. It is a simple but complete model, including a calculable perturbative reheating mechanism that allows one to make definite predictions for the inflationary observables, given a set of input parameters. One testable prediction is that the tensor-to-scalar ratio $r$ is likely to be observable, depending upon the value of the nonminimal coupling of the inflaton to gravity. For the values $\xi \sim 0.01-1$ considered in this work, we have found $r>0.04$, which is within the sensitivity of upcoming $\mathrm{CMB}$ experiments. For example LiteBIRD will probe values down to $r \sim 10^{-3}$ [24].

Since ours is a two-field inflation model, another possible signal is correlated baryon isocurvature-adiabatic fluctuations that have been constrained by the Planck Collaboration. We have found that these can occur at an observable level if the total duration of inflation did not greatly exceed the canonical minimum number of $e$ foldings, $N_{\text {tot }} \sim 60$. In this case the inflaton trajectory can turn significantly in field space around the time of horizon crossing. We are not aware of other models in the literature that predict baryon isocurvature perturbations.

The model relies upon new colored scalar particles in order to transfer the baryon asymmetry from the inflaton to 
the standard model quarks. These could have observable effects in laboratory experiments if sufficiently light, even at the scale of $10^{4} \mathrm{TeV}$ for $K^{0}-\bar{K}^{0}$ oscillations. The colored scalars could also mediate purely hadronic rare flavor-changing decays, that we have not considered here. The new source of baryon violation needed for baryogenesis is however hidden at the high scale the inflaton mass $\sim 10^{-7} m_{P}$, out of reach of laboratory probes.

We have considered only the simplest scenario for reheating. It is possible that sufficiently large values of $\lambda^{\prime \prime}$ could lead to more efficient reheating through parametric resonance [16]. To our knowledge, this has not been previously studied for couplings of the form $\phi \Phi^{3}$ such as are present in our model. Moreover we have ignored the Higgs portal coupling $|\phi|^{2}|H|^{2}$ which could reduce the baryon asymmetry by producing extra radiation. We leave these issues for future study.

\section{ACKNOWLEDGMENTS}

We thank C. Byrnes and R. Namba for very helpful correspondence or discussions. Our work is supported by the Natural Sciences and Engineering Research Council, Canada. M.P. is supported by the Arthur B. McDonald Institute for Canadian astroparticle physics research.
[1] M. Bastero-Gil, A. Berera, R. O. Ramos, and J. G. Rosa, Warm baryogenesis, Phys. Lett. B 712, 425 (2012); Observational implications of mattergenesis during inflation, J. Cosmol. Astropart. Phys. 10 (2014) 053.

[2] I. Affleck and M. Dine, A new mechanism for baryogenesis, Nucl. Phys. B249, 361 (1985).

[3] A. D. Sakharov, Violation of $C P$ invariance, C asymmetry, and baryon asymmetry of the Universe, Pis'ma Zh. Eksp. Teor. Fiz. 5, 32 (1967) [JETP Lett. 5, 24 (1967)]; Usp. Fiz. Nauk 161, 61 (1991) [Sov. Phys. Usp. 34, 392 (1991)].

[4] A. D. Linde, Chaotic inflation, Phys. Lett. 129B, 177 (1983).

[5] Y. Akrami et al. (Planck Collaboration), Planck 2018 results. X. Constraints on inflation, arXiv:1807.06211.

[6] N. Okada, M. U. Rehman, and Q. Shafi, Tensor to scalar ratio in nonminimal $\phi^{4}$ inflation, Phys. Rev. D 82, 043502 (2010).

[7] A. Linde, M. Noorbala, and A. Westphal, Observational consequences of chaotic inflation with nonminimal coupling to gravity, J. Cosmol. Astropart. Phys. 03 (2011) 013.

[8] Y. Y. Charng, D. S. Lee, C. N. Leung, and K. W. Ng, Affleck-Dine baryogenesis, split supersymmetry, and inflation, Phys. Rev. D 80, 063519 (2009).

[9] J. L. Evans, T. Gherghetta, and M. Peloso, Affleck-Dine sneutrino inflation, Phys. Rev. D 92, 021303 (2015).

[10] M. P. Hertzberg and J. Karouby, Generating the observed baryon asymmetry from the inflaton field, Phys. Rev. D 89, 063523 (2014).

[11] N. Takeda, Inflatonic baryogenesis with large tensor mode, Phys. Lett. B 746, 368 (2015).

[12] J. M. Cline, String cosmology, arXiv:hep-th/0612129.

[13] C. T. Byrnes and D. Wands, Curvature and isocurvature perturbations from two-field inflation in a slow-roll expansion, Phys. Rev. D 74, 043529 (2006).
[14] C. Gordon, D. Wands, B. A. Bassett, and R. Maartens, Adiabatic and entropy perturbations from inflation, Phys. Rev. D 63, 023506 (2000).

[15] A. R. Liddle and S. M. Leach, How long before the end of inflation were observable perturbations produced?, Phys. Rev. D 68, 103503 (2003).

[16] L. Kofman, A. D. Linde, and A. A. Starobinsky, Towards the theory of reheating after inflation, Phys. Rev. D 56, 3258 (1997).

[17] J. A. Harvey and M. S. Turner, Cosmological baryon and lepton number in the presence of electroweak fermion number violation, Phys. Rev. D 42, 3344 (1990).

[18] N. Aghanim et al. (Planck Collaboration), Planck 2018 results. VI. Cosmological parameters, arXiv:1807.06209.

[19] A. M. Sirunyan et al. (CMS Collaboration), Search for pair-produced resonances decaying to quark pairs in protonproton collisions at $\sqrt{s}=13 \mathrm{TeV}$, Phys. Rev. D 98, 112014 (2018).

[20] M. Aaboud et al. (ATLAS Collaboration), A search for pair-produced resonances in four-jet final states at $\sqrt{\mathrm{s}}=$ $13 \mathrm{TeV}$ with the ATLAS detector, Eur. Phys. J. C 78, 250 (2018).

[21] C. C. Nishi, Simple derivation of general Fierz-like identities, Am. J. Phys. 73, 1160 (2005).

[22] A. M. Sirunyan et al. (CMS Collaboration), Search for new physics in dijet angular distributions using proton-proton collisions at $\sqrt{s}=13 \mathrm{TeV}$ and constraints on dark matter and other models, Eur. Phys. J. C 78, 789 (2018).

[23] M. Bona (UTfit Collaboration), Latest results for the unitary triangle fit from the UTfit Collaboration, Proc. Sci., CKM2016 (2017) 096.

[24] M. Hazumi et al., LiteBIRD: A satellite for the studies of B-mode polarization and inflation from cosmic background radiation detection, J. Low Temp. Phys. 194, 443 (2019). 